Fizika Nizkikh Temperatur, 1997, v. 23, No 9, p. 923-926

\title{
Peculiarities of domain walls with vertical Bloch lines at low temperatures
}

\author{
V. T. Dovgij, A. A. Kalkin, T. G. Astaf'eva, \\ F. G. Bar'yakhtar, and G. I. Yampolskaya \\ A. Galkin Donetsk Physical and Technical Institute, National Academy of Sciences of the Ukraine, \\ 340114, Donetsk, Ukraine \\ E-mail: sergey@gam.dipt.donetsk.ua
}

Submitted February 29, 1996, revised January 30, 1997

\begin{abstract}
The results of experimental studies of the vertical Bloch line (VBL) generation and stability in the epitaxial iron-garnet films (EIGF) with different compounds, anisotropy, implantation, and compensation temperatures are presented. It is shown that the critical fields of the VBL generation and annihilation in the stripe domain (SD) walls increase and that its values differ considerably (the VBL stability region increases) with decreasing temperature. The domain period increases as a result of magnetic inhomogeneities of the domain walls. The magnetic profile and the energy potential barrier account for the existence of the metastable domain structures with different periods. It is established that the VBL existence domains are limited by the region near the Neel temperature $T_{N}$ and the compensation point temperature $T_{c}$ (in films with $T_{c}$ ).
\end{abstract}

PACS: $75.50 . \mathrm{Bb}, \mathbf{7 6 . 5 0 . + g}$

1. The static and dynamic properties of the VBL in domain walls have been studied extensively (see, for example, Refs. 1-4). Most of these studies were carried out at room temperature and some of them in the range from room temperature to the Néel temperature. The VBL stability in domain walls at low temperatures has been virtually ignored in all studies.

Our main goal was to investigate the $H-T$ diagrams of generation and stability of the VBL at the $\mathrm{SD}$ boundaries and the effect of the easy magnetization axis tilt, orthorhombic anisotropy, compensation point, and the magnetic profile (nonuniformity of the film magnetic properties) on the regions of the VBL stability and generation at low temperatures.

2. The investigation of the VBL creation and stability in the SD boundaries was carried out in the EIGF obtained by the liquid-phase epitaxy on gadolinium-gallium garnet substrata with (111) orientation. The magnetic parameters of the film have been measured by the methods suggested in Refs. 5-7 and are presented in Table 1. The investigations were carried out using magnetooptic devices with the Faraday effect. The creation of straightforward domain walls with the VBL was carried out by spontaneous demagnetization of films at the current pulse in the current loop (two parallel applications with $30 \mu \mathrm{m}$ diameter, the distance between conductors is $300 \mu \mathrm{m}$ ). The current pulse in the loop with the amplitude is $I_{\max }=12 \mathrm{~A}$, the pulse time is $\tau_{p}=0.2-10 \mu \mathrm{s}$, and the front slope of the increase and decay pulse up to $\tau_{f}=50 \mathrm{~ns}$ was created by the pulse magnetic field with amplitude $H_{\max }=200 \mathrm{Oe}$ directed along the easy magnetization axis of the sample and is essential for film saturation. In this way the stripe structure with domains perpendicular to the conductors was created. The wall hardness was defined by the collapse field of the bubble domains obtained by the SD cutting by means of the bias current short pulse [8]. The impulse amplitude was minimally possible in order for the additional VBL not to be generated.

3. The typical $H-T$ diagram of the VBL stability for the $(\mathrm{YSmLuCa})_{3}(\mathrm{FeGe})_{5} \mathrm{O}_{12}$ film (film 1 in Table 1), which has a small tilt of the magnetization easy axis, and the orthorhombic anisotropy are presented on Fig. 1. Line 1 is the limit of the region of the VBL «static» stability (or the line of the VBL annihilation). The shaded region corresponds to the stable VBL existence in the SD boundaries. For determination of this line after spontaneous 


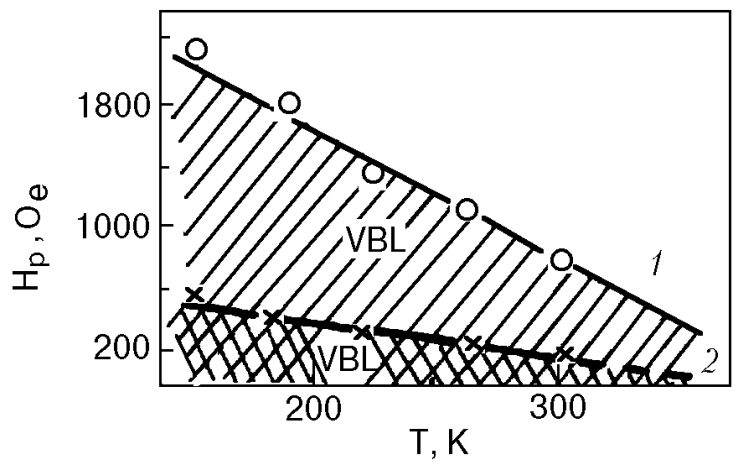

Fig. 1. The «static» and «dynamic» $H-T$ diagrams of the VBL stability in the SD boundaries (the VBL annihilation and generation $H-T$ diagram).

demagnetization of the film, the SD structure was influenced by the planar quasistatic field $H_{p}$, which was perpendicular to magnetization easy axis and parallel to the film plane. The VBL existence was then determined by the dispersing of the bubble domain collapse field. It is shown that the critical field $H_{c r}$ (the VBL annihilation field), above which the hard bubble domain is not created, increases with decreasing temperature. Thus, each temperature has the corresponding value of the planar critical field $H_{\text {cr }}$ (when the VBL are not created) and the critical temperature exists when the $H_{\mathrm{cr}}$ vanishes. The considerable increase of $H_{\mathrm{cr}}$ with decreasing temperature results primarily from the uniaxial anisotropy increase.

Line 2 in Fig. 1 corresponds to the limit of the VBL «dynamic» stability (or the VBL creation line). In order to determine this line, the quasistatic planar field $H_{0}$ must influence EIGF during its spontaneous demagnetization. After that the VBL presence in the SD boundaries was determined. Line 2 corresponds to the planar field value higher than the spontaneous which takes place without the VBL creation. The touched region corresponds to the stable VBL creation region.

It is shown that the critical planar field of the VBL annihilation in stripe domains is essentially

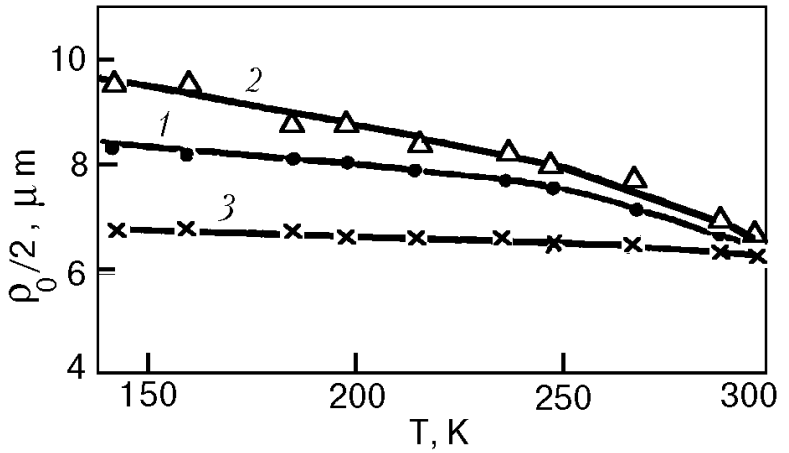

Fig. 2. Temperature dependence of the SD period for different generation methods in EIGF. Lines correspond to: 1 - The method of the displacement field with low-frequency modulation; 2 - spontaneous demagnetization method; 3 - method of the quasi-static plane field.

higher a the critical planar field that prevents the VBL creation. The «static» region of the VBL stable existence is essentially greater then the «dynamic» region. The experimental values of the static and dynamic critical fields $H_{\text {cr }}$ exceed the theoretical value $8 M$. Such great difference can be explained by the existence of magnetic profile in the film [9]. The profile causes the existence of the high potential barrier, which prevents the VBL annihilation.

The SD period-vs-temperature curves for various methods of the SD creation and the hysteresis of the SD period dependence from planar field value for the same film acknowledge the existence of the profile and the metastable states. In Fig. 2 line 1 characterizes the temperature dependence of the SD structure period created in the direct-displacement field with the alternating field. Curve 2 corresponds to the SD system created with the EIGF spontaneous demagnetization. Curve 3 corresponds to the SD structure formed by the quasistatic planar field. Line 1 describes the equilibrium structure, because domain structures 2 and 3 were transformed into first one after the influence of the pulse or alternating displacement field. We see that curve 2 is situated above line 1 and their difference increases

Table 1

The compounds and parameters of epitaxial iron-garnet films: $h$ is the sample thickness, $P_{0}$ is the SD period at zero field, $4 \pi M$ is the saturation magnetization, $H_{a}$ is the uniaxial anisotropy field, and $A$ is the constant of inhomogeneous exchange interaction

\begin{tabular}{c|c|c|c|c|c|c}
\hline \hline & Compound & $h, \mu m$ & $P_{0}, \mu m$ & $4 \pi M, \mathrm{Gs}$ & $H_{a}, \mathrm{Oe}$ & $A \cdot 10^{-7}, \mathrm{erg} / \mathrm{cm}$ \\
\hline \hline 1 & $(\mathrm{YSmLuCa})_{3}(\mathrm{FeGe})_{5} \mathrm{O}_{12}$ & 6.8 & 13.8 & 183 & 1840 & 2.0 \\
2 & $(\mathrm{YSmCa})_{3}(\mathrm{FeGe})_{5} \mathrm{O}_{12}$ & 6.8 & 9.9 & 203 & 1420 & 4.0 \\
3 & $(\mathrm{YBi})_{3}(\mathrm{FeGa})_{5} \mathrm{O}_{12}$ & 6.9 & 8.5 & 233 & 4300 & 2.7 \\
4 & $(\mathrm{YGdTm})_{3}(\mathrm{FeGa})_{5} \mathrm{O}_{12}$ & 9.0 & 18.4 & 161 & 1580 & 2.9 \\
\hline \hline
\end{tabular}




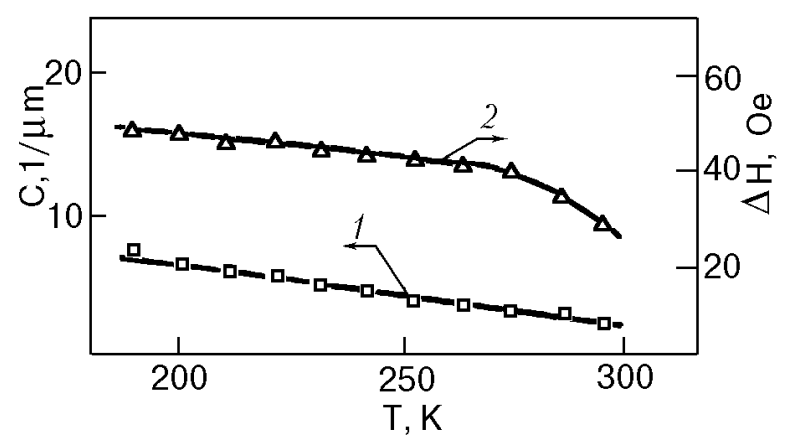

Fig. 3. Temperature dependences of the VBL density and the bubble domain collapse fields.

with the temperature decrease. The equilibrium SD structure arises with the period determined by the dynamic energy of the domain boundaries and the demagnetization fields because the spontaneous demagnetization takes place without the external magnetic field (process develops after the end of current pulse). Thus, we can assume that the SD period increase is conditioned by the domain structure energy increase. The Bloch lines are one of the sources of this increase.

The density of the mean energy of the domain structure with the VBL can be determined by the expression [1]

$$
\sigma=\sigma_{0}\left[1+\left(\pi \Delta_{0} / s\right)^{2}+(2 Q)^{-1}\right]^{1 / 2},
$$

where $K_{u}$ is the uniaxial anisotropy constant; $Q=H_{A} / 4 \pi M_{s}$ is the quality factor; $\pi \Delta_{0}=$ $=\pi\left(A / K_{u}\right)^{1 / 2} ; \sigma_{0}=4\left(A K_{u}\right)^{1 / 2}$ are the thickness and the energy density of the Bloch-type domain wall, and $s$ is the distance between the VBLs. The VBL density in domain walls on spontaneous demagnetization can be determined by means of a theory which assuming that line 1 corresponds to the domain structure without VBL.

Line 1 in Fig. 3 is the dependence of the VBL density from temperature and line 2 characterizes the temperature dependence of the dispersion of the bubble domain collapse fields. Experiment shows that the domain walls have the high density of the VBL through spontaneous demagnetization. It leads to an increase in the domain structure period. The investigation of domain structures formed by the direct and alternating displacement fields has shown that the domain walls have very low density of Bloch lines (see line 1 in Fig. 2). As can be seen from Fig. 2, the period of the domain structures formed by the quasistatic plane magnetic field is almost independent of temperature. But it is always smaller than the equilibrium domain structure period and this difference increases with decreasing

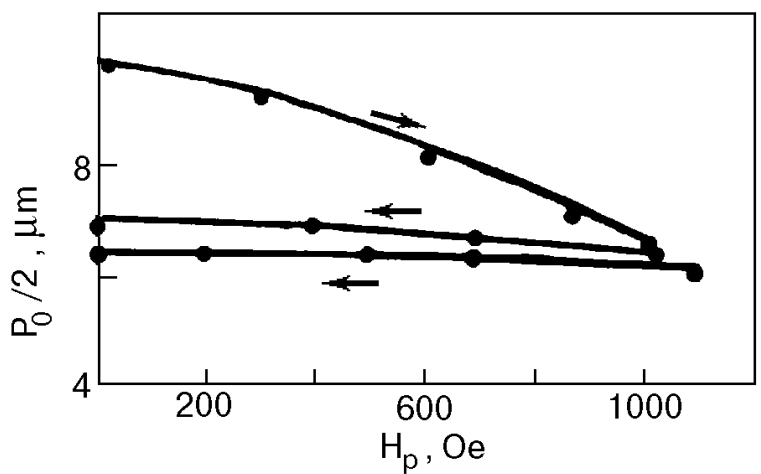

Fig. 4. The hysteresis of dependence of the SD period from the plane field value.

temperature. The decrease of the domain structure period can be explained by the potential barrier between the most equilibrium state of the boundary and other metastable states, as well as by the influence of the plane field. It is known [10] that the energy of domain wall decreases more quickly with increasing field than the demagnetization energy. It leads to a decrease in the domain structure period. The domain structure is frozen in one of the metastable states by a decrease in the plane field. The temperature is lower, the energy barrier is higher, and the difference in the periods from the equilibrium value is greater. The SD period-vs.plane field dependence is shown in Fig. 4. The direction of the plane field change is indicated by the arrows. The period-vs.-plane field dependence was measured at the fixed temperature of $133 \mathrm{~K}$. The upper curve contains the large VBL quantity and corresponds to line 2 in Fig. 2. The lower curve contains the small VBL quantity and corresponds to line 3 in Fig. 2. Thus, the magnetic microprofile and the energy potential barrier account for the existence of the metastable states with different dimensions at the same temperature.

4. The same dependences of the SD period as a function of temperature for different methods of creation was measured in an implanted $(\mathrm{YSmCa})_{3}(\mathrm{FeGe})_{5} \mathrm{O}_{12}$ film (see Fig. 5). In this film the collapse field dispersion $\Delta H$ is not revealed. This fact can be explained in the following way. During spontaneous demagnetization of EIGF the Bloch lines or loops are generating in the SD walls. They change the dynamic energy of the domain walls and increase the SD period. After termination of the film demagnetization the inhomogeneities disappear because they are unstable in the static state and the SD period does not changed because of the high potential barrier which prevents the domain generation at low temperatures.

Thus, the Bloch line generation and existence lead to an increase in the domain structure period at low 


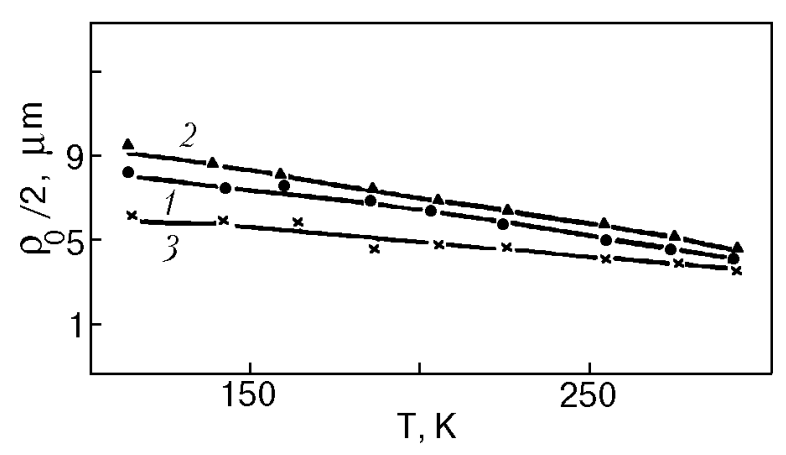

Fig. 5. Temperature dependence of the SD period for different generation methods in implanting EIGF. Lines 1, 2, and 3 are the same as those in Fig. 2.

temperatures. The magnetic profile and the energy potential barrier lead to the existence of metastable domain structures with different periods and increase the VBL generation and stability region with decreasing temperature.

5. The influence of the easy-axis tilt and orthorhombic anisotropy is illustrated in Fig. 6 for the (110) orientation $(\mathrm{YBi})_{3}(\mathrm{FeGa})_{5} \mathrm{O}_{12}$ film as the temperature is lowered. We see that the critical field of the VBL generation decreases with decreasing temperature, in contrast with the $H-T$ diagrams in Fig. 1 and the $d H / d T$ derivative is different for in-plane film hard axis and easy-axis. Thus, the increase of the easy axis tilt and orthorhombic anisotropy leads to a change in the VBL generation and existence domain as the temperature is lowered.

The presence of the compensation point $T_{c}$ in the film leads to cutting of the VBL existence domain near the $T_{c}$. The VBL generation and existence domain is shown in Fig. 7 for the $(\mathrm{YGdTm})_{3}(\mathrm{FeGa})_{5} \mathrm{O}_{12}$ film with $T_{c}=210 \mathrm{~K}$. This

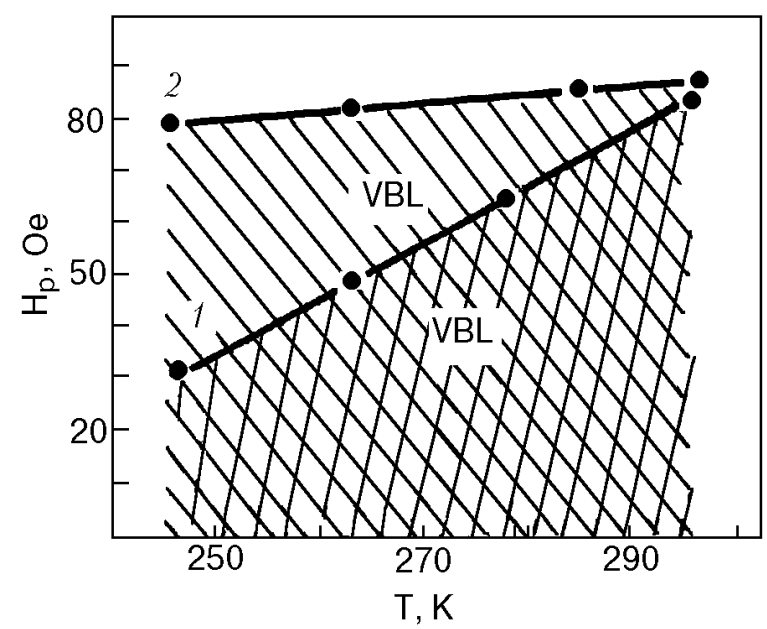

Fig. 6. The $H-T$ diagram of the VBL stability in the SD boundaries of the $(\mathrm{YBi})_{3}(\mathrm{FeGe})_{5} \mathrm{O}_{12}$ film. Line 1 corresponds to the easy axis; line 2 corresponds to the hard axis.

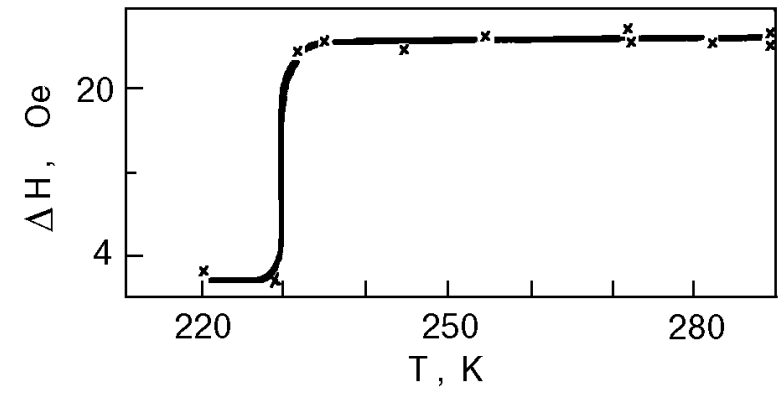

Fig. 7. Temperature dependence of the bubble domain collapse field dispersion for the EIGF with the compensation point $T_{c}$.

effect is connected with the magnetization decrease on approaching the compensation temperature and with the decrease in the demagnetizing field by the EIGF spontaneous demagnetization (a small quantity of the VBL is generated). For investigating EIGF with $T_{c}$ the domain of the VBL stable existence is limited by the Neel temperature $T_{N}$ and the compensation temperature $T_{c}$ with some deviation $\Delta T$ from these critical points. Usually, $\Delta T$ is in the range $20-30 \mathrm{~K}$.

6. Thus, the increase of the plane critical fields of the VBL generation and annihilation in the SD boundaries, as well as the considerable difference in its values takes place at low temperatures. The magnetic inhomogeneities in the domain walls cause the domain period to increase. The magnetic profile and the potential barrier existence lead to the existence of the metastable domain structures with different periods. The easy-axis tilt, the orthorhombic anisotropy, and the compensation point existence lead to a decrease of the VBL generation and stability region in the EIGF.

1. A. P. Malosemoff and J. C. Slonczewski, Magnetic domain walls in bubble materials, Mir, Moscow (1982).

2. A. Eschenfelder, Magnetic bubble technology, Mir, Moscow (1983).

3. T. O'Dell, Ferromagnetodynamics. The dynamics of magnetic bubbles, domains, and domain walls, Mir, Moscow (1983).

4. L. S. Uspenskaya and V. K. Vlasko-Vlasov, Zh. Eksp. Teor. Fiz. 101, 944 (1992).

5. R. F. Shaw, D. E. Hill, R. M. Sandford, and J. M. Moody, J. Appl. Phys. 44, 2346 (1973).

6. W. F. Druyvestein, F. W. Dorleijn, and R. F. Rinierse, $J$. Appl. Phys. 44, 2397 (1973).

7. A. Hubert, A. P. Malozemoff, and J. C. De Luca, J. Appl. Phys. 45, 3562 (1974).

8. H. Nishida, T. Kobayashi, and Y. Sugita, IEEE Trans. Magn. MAG-9, 517 (1973).

9. V. T. Dovgij and A. A. Kalkin, Pis'ma Zh. Techn. Fiz. 15, 89 (1989).

10. T. R. Johunsen, D. J. Norman, and E. J. Toroh, J. Appl. Phys. 42, 1715 (1971). 